\title{
El proceso de reforma de la Administración en Italia ${ }^{1}$
}

Bruno Dente*

\section{Introducción: las reformas administrativas de la XI Legislatura}

Es hasta cierto punto paradójico, pero no por ello menos cierto, que la más breve y las más atormentada de las legislaturas de la Italia republicana (aunque la XII puede superarla en brevedad y ya está resultando más atormentada) haya sido la que más ha colocado el tema de la "reforma del Estado" (o sea, la transformación del modo de ser y de actuar de las Administraciones públicas) en el centro del debate político, social y periodístico. Ello ha sido así como nunca en el pasado, y no sólo porque nuevos proyectos se iban añadiendo a los muchos ya existentes, sino sobre todo porque algunas transformaciones, no precisamente desdeñables, veían finalmente la luz.

La "cuestión administrativa", a menudo citada en los debates televisivos, pero nunca puesta de forma efectiva en el centro de la atención gubernamental, ha logrado, en esos dos años que van de 1992 a 1994, alcanzar un punto de inflexión. En el sentido, que ha parecido, quizás por vez primera, que se ponía en marcha un proceso irreversible para la modernización de la Administración pública, o lo que es lo mismo, se confirmasen las esperanzas de que la Administración pública italiana pudiera perder las características de excepcionalidad negativa que la vox populi, con todos los motivos, le atribuía.

Más allá de los juicios que se irán formulando en la descripción de los distintos episodios de reforma y también relativamente más allá de la caracterización de conjunto del proceso de reforma, parecen indiscutibles dos puntos:

1. La cuestión de la reforma de la Administración pública ha tenido en el bienio 1992-1994 una presencia en los medios de comunicación absolutamente inusitada; un solo indicador nos puede servir: en el año en el que el ministro Cassese ha ejercido como tal, el periódico Sole 24 Ore (que es el principal periódico económico italiano) lo ha citado 630 veces, mientras su predecesor, Gaspari, lo fue 320 veces, en un período de tiempo comparable.

2. El número de procedimientos normativos y, más en general, de iniciativas asumidas por el Ministerio para la Función Pública y por el Gobierno en materia de reforma de la Administración fue ciertamente impresionante. En casi todos los posibles terrenos de intervención tradicional, y sobre muchos no tan tradicionales, podemos reseñar actuaciones, innovaciones, intervenciones.

Es por lo tanto imprescindible intentar hacer un balance del pasado reciente, ya que ello nos puede ayudar a fijar la agenda para el futuro próximo.

Creo que en un punto, tanto los observadores como los propios operadores que conocen bien la Administración pública italiana concuerdan plenamente: al margen de su deseabilidad, las transformaciones de la forma de Estado, por ejemplo, en sentido federalista, y de la forma de gobierno, por ejemplo, en un sentido presidencialista, corren el riesgo de no alcanzar los efectos deseados si al mismo tiempo no se modifican los mecanismos de funcionamiento de la Administración pública. De hecho, se puede argumentar fácilmente que la reforma regionalista de los años 70 ha fracasado, o al menos no ha logrado los efectos esperados, precisamente en el terreno de la maquinaria administrativa. En realidad, podemos ir bastante más allá: como muy bien ha indicado Marco Cammelu, desde una perspectiva comparada, el auténtico hándicap de Italia en el mercado internacional ha residido en su Administración pública obsoleta, ineficiente, autorreferencial. El proceso de modernización, por tanto, no es un lujo, o un aspecto entre otros muchos de esta transición difícil, se trata de un elemento previo a cual- 
quier juicio, ya que citando nuevamente a Cammelu, "una Administración fuertemente degradada...pone en cuestión las propias raíces de legitimidad de las instituciones, sea cual sea la estructura corstitucional o la mayoría de gobierno".

De aquí, la necesidad de realizar un balance del período apenas dejado atrás, aunque se trate de un balance provisional o parcial, ya que en muchos casos la verdadera relevancia de algunas de las reformas introducidas sólo será posible verificarla a medio y largo plazo. Y de aquí, el que ello implique no sólo documentar las reformas introducidas, o propuestas, sino entrar directarn ente en su mérito para preguntarse:

1. ¿Hasta qué punto y en qué medida esas reformas y propuestas constituyen realmente una novedad o, utilizando la expresión de Giovanni COSTA, expresan un momento de discontinuidad?

2. ¿Hasta qué punto y en qué medida son congruentes con algunas tendencias de fondo de transformación del sistema, y especialmente con la tendencia a la descentralización?

3. ¿Hasta qué punto y en qué medida esas reformas y propuestas son al menos potencialmente irreversibles, en el sentido de incorporar mecanismos de autoalimentación?

Juntc: a este proceso de evaluación, resulta oportuno preguntarse también si podemos leer la transformación italiana sólo desde el interior de la difícil transición política e institucional que est:_nos atravesando, o si más bien debemos referirla a un procesc de alcance más general que se relaciona con algunas modific iones en absoluto marginales que conectan la sociedad $\mathrm{y}$ las in: ituciones.

Sobı : este último punto volveré más adelante y en las conclusion s intentaré ofrecer algunas respuestas generales y problemati ts acerca de las preguntas antes formuladas, que tratan de enju ciar el programa político de modernización de la Administración pública que se desplegó en la XI Legislatura.

Antes conviene plantearse otras cuestiones, interrogándose sobre los factores que han consentido el desarrollo de esa voluntad y proyectualidad reformadora. Admitiendo que todo lo que ha ocurrido en la XI Legislatura en el campo de la reforma administrativa deba explicarse por la propia excepcionalidad del momento (lo que resulta dificilmente contestable si atendemos a lo ya mencionado en relación a la atención dispensada por los media), deberíamos preguntarnos qué factores explican la repentina priorización de eso que en el pasado se denominaban "las ramas bajas" de la reforma institucional. Personalmente creo, como ya he dicho, que estas "ramas bajas" son en realidad las auténticas raíces del árbol que representa la constitución material de nuestro país, de tal manera, que el sistema electoral, los mecanismos de formación y de composición de los órganos gubernamentales y el mismo reparto de las competencias entre centro y periferia, y la eficacia de todos ellos, deriva en gran parte del buen funcionamiento de los mecanismos de gestión de los servicios públicos y más en general, del establecimiento de una relación positiva entre Administración y administrados. Precisamente, por todo ello, el núcleo de esta contribución pretende ser la identificación de los factores de éxito de las reformas administrativas de nuestro reciente pasado, para extraer de los mismos, indicaciones y elementos de aprendizaje que nos sirvan para el nuevo período que se avecina.

Antes, no obstante, es preciso caracterizar mejor lo ocurrido entre 1992 y 1994 y en particular preguntarse si los dos gobiernos que han formado la XI Legislatura, y que aunque en medida diferente, han puesto en un lugar central la reforma administrativa, han mantenido posturas y formas de operar similares, o bien distintas e incluso opuestas.

\section{Las fases del proceso de reforma}

En realidad, la posibilidad de que los procesos de transformación puestos en marcha en el período del Gobierno Ciampi y bajo el impulso del ministro Cassese fueran completamente diversos e incluso opuestos a los que inició el gobierno Amato bajo la dirección del subsecretario Sacconi, es sumamente improbable. La historia intelectual de los protagonistas, y su participación activa en los debates y las reformas de los últimos treinta años garantizan que, por encima de los acentes personales de uno u y otro, ellos forman parte de una misma policy community ${ }^{2}$, y comparten muchas de las orientaciones de fondo.

La caracterización de las dos fases que, en ciertos casos, como veremos, funciona bastante bien, se basa inevitablemente en un cierto forzamiento, en el sentido en que se eliminan los factores que forman el sustrato común, y se resaltan los factores residuales, que quizás son minoritarios. Por otra parte, conviene recordar que el principal protagonista de la segunda fase, es decir el ministro Sabino Cassese, ha estado muy crítico con algunas de las principales decisiones de la fase precedente $e^{3}$, sobre todo en relación a la privatización del empleo público creada por el Decreto Ley 29/93 (DL 29/93). Por ello, podemos decir, que las diferencias entre ambos períodos vendrían legitimadas de alguna forma por el comportamiento de sus propios protagonistas.

El esquema de análisis que utilizaré para caracterizar las distintas fases se basa más en el modo de ser y de actuar del gobierno, que en condiciones del entorno, como por ejemplo las constelaciones de actores relevantes, o la posible importancia atribuida a la puesta en práctica, que no me parece especialmente diferente en uno y otro caso (pero, sobre este punto veáse, no obstante, el párrafo sucesivo).

El primer elemento que resulta relevante es el tipo de aproximación utilizada, es decir, el modo a través del cual se organizó la comunicación externa de las iniciativas gubernamentales. En este aspecto, creo que se puede comprobar una diferencia bastante clara. Durante el gobierno Amato la reforma administra- 
tiva se presentó esencialmente a través de la propuesta de dos slogans o si se prefiere de dos principios generales que querían mostrar la novedad de las reformas propuestas y realizadas. El primero de estos slogans fue indudablemente la realización de la separación entre politica y Administración, que en realidad alude a la cuestión de la crisis del sistema político italiano. La segunda hace referencia a la oportunidad de avanzar en una privatización de la relación laboral del empleo público, lo que permitía al mismo tiempo satisfacer las tendencias hacia la desregulación y la reducción del sector público muy presente en la sociedad italiana (como se demostró rápidamente en los resultados electorales), y la realización de un objetivo claro del sindicato, es decir, la completa contractualización de las relaciones industriales en este importante sector laboral.

La organización de la comunicación en torno a estos dos principios denota una aproximación que podríamos definir como sintética, en el sentido que busca concentrarse en pocos principios generales de los que deducir las concretas operaciones de reforma y también de carácter simbólico, al menos en la medida en que ni la separación entre política y administración, ni la privatización de las relaciones laborales llegaron a ver la luz, sino por otros motivos, porque eran demasiadas y demasiado diferentes tales principios y las realidades administrativas a las que tales principios debían aplicarse. Eso no significa, obviamente, que esa aproximación no fuera en absoluto efectiva: la organización de la comunicación en torno a estas dos cuestiones, que corresponden a reinvidicaciones históricas de los directivos públicos y del movimiento sindical, permite abarcar estratos amplios de la máquina administrativa y poner en marcha un amplio debate sobre los objetivos y sobre la filosofía de la reforma. Pero sobre esto volveremos en seguida. Lo que ahora conviene subrayar es que el enfoque del Gobierno Ciampi y de Sabino Cassese especialmente ha sido completamente diferente.

Las críticas que como estudioso realizó CASSESE al DL 29/93 subrayaban como no era posible tratar el amplio cuerpo de la Administración pública italiana como un todo único. El mismo juicio se desarrolla en lo que constituye el manifiesto de su ministerio, Indirizzi per la Modernizzazione, que no por casualidad concluye con un decálogo de cosas a hacer que afectan de forma simultánea a muchos planos diferentes. Pero probablemente es aún más significativo, que la primera realización concreta llevada a cabo se caracterice por su carácter cognoscitivo sobre una multiplicidad de problemas. Me refiero al Informe sobre las condiciones de las Administraciones públicas realizado en pocas semanas, con el objetivo claro de proporcionar el diagnóstico, o los diagnósticos de los males que aquejaban a nuestra maquinaria pública. CASSESE se convierte en intérprete de un enfoque analítico en relación a la reforma, que pone de relieve la imposibilidad de basarse en pocos princpios claros, sino que subraya en cambio la exigencia de realizar un catálogo de los problemas y de las soluciones disponibles para cada uno de ellos. De alguna manera, el gran riesgo del Gobierno Ciampi era el contrario del Gobierno Amato; si éste último corría el riesgo de que no se tomaran en serio sus intentos de reforma, más que nada por lo abstracto de sus enunciados, y por el hecho de sus excesivas excepciones, CASSESE corría el riesgo de que no se comprendiera el significado innovativo de su propuestas, por la falta de una identificación simbólica más de conjunto.

La verdad es que ha habido intentos en este sentido (poner al ciudadano en el centro, por ejemplo), pero un índice de las dificultades que encuentra un enfoque analítico en un país como el nuestro lleno de retórica, es el episodio de la gran indignación que despertó por un simple estudio que simulaba por extrapolación el número de puestos de trabajo que se podían crear en el sector público italiano en los próximos años, y que se interpretó de forma instrumental (pero no por ello menos eficaz) que lo que se buscaba era el proseguir la expansión del sector público. En realidad, el riesgo no se corrió ya que la vida del gobierno Amato, al margen del tema del DL 29/93, fue muy breve, y porque, como se podría decir con un poco de provocación, la identificación simbólica y de conjunto del Gobierno Ciampi fue en realidad la propia figura de CASSESE, presentándose como garantía e intérprete, por sus características personales ampliamente reconocidas incluso por sus opositores, de una clara voluntad de renovación. Pero, ciertamente, el problema de la relación entre reforma y reformas se mantiene como uno de los elementos distintivos de la dos fases que acabamos de presentar.

Pero, prosiguiendo el análisis, más allá del enfoque general utilizado, ise puede hablar en las dos fases de una filosofia diferente sobre el tipo de intervención a realizar, o dicho de otra manera, de una acentuación de principios que podamos considerar como alternativos? Es éste el campo en el que el análisis resulta quizás más forzado, al menos porque, como ya hemos apuntado, los elementos de continuidad tienden a prevalecer sobre los elementos de discontinuidad. Aclarado esto, no es realmente difícil caracterizar la filosofía dominante durante el período Amato/Sacconi como de neo-tradicional, sin que ello implique un juicio negativo. Para aclarar más qué entiendo con esta expresión, me referiré a la ley $241 / 90$, una ley que lleva a cabo una vieja aspiración de la doctrina italiana: la articulación general del procedimiento administrativo. Y lo hace a través del reconocimiento de una serie de derechos a los administrados que pretenden facilitar la exigencia de unos mecanismos más previsibles y más transparentes de formación de la voluntad pública. Se trata de una ley sin duda innovadora (más, a mi juicio, que la ley 142/90, de reforma del gobierno local), pero se inserta de pleno en la tradición secular de contención de la posible arbitrariedad de la autoridad y de la correlativa garantía de la libertad de los ciudadanos. Es esta misma filosofía la que llena enteramente la obra de gobierno del Gobierno Amato, que se coloca, quizás con mayor decisión, en una posición de continuidad sustancial con la evolución del sistema administrativo.

¿Se puede aplicar el mismo juicio a la fase Ciampi/Cassese? Desde una perspectiva amplia, creo que debe responderse a la pregunta de forma afirmativa, tanto por lo ya dicho como por lo que diremos más adelante. Y, a pesar de todo, aunque sea forzando más allá de lo lícito nuestro análisis, en este segundo período emergen una serie de elementos de superación de la filosofia neo-tradicional. Así, se abordan temas nuevos: el len- 
guaje de la Administración, y no sólo en su relación con los ciudadanos (Código de Estilo) o la ética administrativa (con el código de comportamiento de los funcionarios públicos). En segundo lugar, y también en la relación entre Administraciones y ciudadanos y entre Administraciones y ley, se dan innovaciones de base. Pensemos, por ejemplo, en la potencial contractualización de las relaciones entre prestadores y usuarios de servicios públicos, que encontramos de forma implícita en la adopción de las llamadas cartas de servicios. Y pensamos, sobre todo, en el cambio que se opera en la modificación del art. 19 de la ley 1241/90, a través de la transformación del viejo principio del silencio negativo que encamina a una potencial desregulación de las actividades privadas, o la norma, contenida en las correcciones al DL 29/93, según la cual los nuevos contratos colectivos derogan las disposiciones legislativas realizadas con posterioridad a la entrada en vigor del contrato precedente. En tercer lugar, la filosofía de la descentralización sufre importantes aceleraciones, como por ejemplo en lo que concierne a la atribución de los presupuestos de las Universidades. No quisiera extenderme sobre ello, pero creo que se pueda concluir que, con todas las cautelas necesarias, durante el Gobierno Ciampi, se avanzó en una filosofía diferente, no tradicional y potencialmente destructiva de algunos principios básicos de nuestra Administración pública, filosofía que puede de algún modo contraponerse a la orientación neo-tradicional del Gobierno Amato.

Resulta más fácil caracterizar los objetivos perseguidos en cada una de las dos fases que aquí analizamos. Aquí lo que está en juego no son los principios inspiradores o los modos de operar o de presentar las acciones, sino la finalidad con que se abordan las intervenciones. En el caso del Gobierno Ciampi, se trata de un punto muy claro y que se ratifica más de una vez: más allá de las implicaciones de orden general o de renovación institucional, todo el proceso de modernización de la Administración pretendía contener el gasto público, a través de la introducción del cálculo económico en la Administración pública. La consideración de la reforma administrativa como vinculada a los problemas de las finanzas públicas constituyó una de las ideas-fuerza del ministerio Cassese. Así, todo el debate de los presupuestos del 94 y las ásperas discusiones que generó giraron sobre la eficacia de las medidas de ahorro que se planteaban. En realidad, ello era también así en la primera fase del Gobierno Amato, ya que la ley 421/92 que contenía la delegación legislativa al Gobierno en relación a temas de reforma del empleo público ha estado aprobada en el marco de la gigantesca maniobra financiera que siguió a la devaluación de 1992. También, por tanto, la exigencia de resanar las cuentas del Estado ha constituido para el Gobierno Amato el tren en el que hacer subir los ajustes estructurales de la maquinaria pública. Pero, en el momento de la emisión del DL 29/93 (como también el DL que instituyó la Autoridad para la Informática, o el DL sobre reforma de la Corte dei Conti) las exigencias de ahorro habían sido satisfechas de otra forma, esencialmente con el aumento de la presión fiscal y la reducción de las prestaciones sociales, y, por tanto, los objetivos en las Administraciones públicas eran otros: una racionalización general de la actividad gubernamental, y también la garantía del consenso social a través de la implicación de las fuerzas sindicales. Si en el primero de estos objetivos existe continuidad a lo largo de la XI Legislatura (e incluso con todas las anteriores legislaturas, si hacemos caso al menos de sus declaraciones e intenciones), en el segundo me parece que existe una cesura. El acuerdo con los sindicatos no fue una de las principales prioridades del ministro Cassese, ni mucho menos el criterio para evaluar la bondad de las medidas tomadas, de la misma forma como la contención del gasto público no era reivindicado por sus predecesores cuando presentaban los contenidos y los mecanismos de las reformas normativas.

El último elemento considerado en este esquema de análisis que hemos utilizado para caracterizar las dos fases de la reforma se relaciona con el modus operandi de los Gobiernos Amato y Ciampi. ¿Podemos decir, con todas las salvaguardias que el nivel de generalización de la pregunta impone, que existieron diferencias significativas entre ambos? Creo que sí, y pienso que la diferencia en este caso es evidente. ¿Cómo realizó Amato algunas de sus reformas principales? A través de la creación de aparatos 0 mecanismos nuevos o específicos, con la misión de traducir en actividades concretas las orientaciones reformadoras. Tanto si se trata de la Agencia para los representantes como instrumento para la contractualización de las relaciones laborales, o se trate de la Autoridad para la Informática para dar un nuevo impulso a la automatización administrativa, o del Gabinete de las relaciones con el público para la implementación de la ley 1241/90, el significado general no se modifica. De forma coherente con el enfoque neo-tradicional ya mencionado, la búsqueda de nuevos objetivos o el desarrollo de nuevos servicios se garantiza a través de la creación de nuevos aparatos o de nuevas articulaciones organizativas. También se modifican las estructuras precedentes, pero los aspectos más precisos de la reforma se confían a nuevas estructuras que se añaden a las ya muchas agencias, observatorios o autoridades creadas en el pasado. Se trata por tanto de una operatividad por adición que, ante la restricción del corto plazo y la imposibilidad de macrotransformaciones organizativas, pone el acento en la urgencia de crear sujetos adecuados a las nuevas tareas a desarrollar.

La mismas limitaciones temporales no impidieron al Gobierno Ciampi y al ministerio Cassese elaborar un proyecto de reordenación general de la Administración del Estado, que finalmente no se adoptó por resistencias básicamente de tipo político, y ahí existía también, aunque en medida menor, un recurso a la adición organizativa. Pero lo que caracteriza de forma clarísima la orientación de Cassese es el sentido contrario a la adición. Su orientación es la de simplificación de la estructura organizativa mediante un mecanismo de eliminación de aparatos, órganos, funciones y fases procedimentales. De alguna manera, la fase Cassese puede caracterizarse como la de una operatividad por reducción, que se configura como abolición de ministerios, comités interministeriales, órganos colegiados, fases procedimentales (en primer lugar, la fase de control previo de la Corte dei Conti). Se tiene la impresión de que la falta de tiempo haya llevado a direcciones contrarias: por un lado, a poner el acento en 
la prioridad de las actuaciones y sobre la exigencia de hacerlas depender de sujetos organizativos a quien responsabilizar de las mismas, mientras, por el otro lado, se enfatizaba la oportunidad, quizás única, que ofrecía la crisis política e institucional italiana, en el sentido de permitir superar las resistencias a la simplificación de los aparatos administrativos y la posibilidad de repensar los mecanismos de coordinación y de control que fueron estratificándose a lo largo del tiempo.

Resumiendo, diríamos que podemos caracterizar las intervenciones de los gobiernos Amato y Ciampi, como mancomunados en la importancia atribuida a la reforma administrativa y en algunas orientaciones de fondo, pero diferentes por los enfoques (sintético/simbólico el primero, analítico el segundo), por filosofías (neo-tradicional o con elementos innovadores), por los objetivos (de racionalización y consenso social o de contención del gasto público) y por el modus operandi (una operatividad por adición en el primer caso, y una operatividad por reducción en el segundo).

\section{Factores de éxito}

Hemos llegado así a afrontar lo que constituye nuestro centro de atención, es decir, la identificación de los elementos que han consentido el desarrollo del proceso de reforma, o dicho de otra manera, los factores de éxito que han contribuido a poner en el centro de la atención pública la cuestión administrativa y, sobre todo, que han permitido introducir significativos elementos de novedad en nuestro panorama institucional.

La investigación realizada no pretende, evidentemente, ser exhaustiva. Ante todo porque sería necesario un trabajo imposible de reconstrucción de los varios centenares de procesos decisionales en los cuales se ha articulado ese período de reforma que hemos atravesado. Una de las diferencias principales que se pueden destacar entre la XI Legislatura y los períodos precedentes es que no existe un texto legislativo que pueda caracterizar, aunque sea a efectos simbólicos, todo el proceso. El texto más significativo desde este punto de vista es el DL 29/93, pero no puede asumir ese carácter simbólico, porque ha estado reformado dos veces y porque ha sido uno de los puntos de controversia en el paso de la primera a la segunda fase. Podríamos decir que, de hecho, lo que caracteriza el reformismo administrativo de la Legislatura es precisamente el hecho de la falta de identificación de la reforma con un aspecto específico, por importante o estratégico que pueda parecer. No es precisamente uno de los menores méritos del ministerio Cassese el mostrar que para ser eficaces en el terreno de las transformaciones de los aparatos públicos no puedes confiar en un instrumento solo, por general o relevante que sea. En este sentido, el primero de los factores de éxito que estamos buscando y que es uno de los elementos más positivos de todo el período reformador, es la renuncia al carácter "sinóptico" de la reforma. La capacidad de segmentar el problema, y no la investigación de una imposible piedra filosofal capaz de transformar en oro el material poco valorado del que parece estar hecha nuestra Administración pública es, en efecto, al mismo tiempo una condición de eficacia y la explicación de los logros conseguidos.

Este último elemento introduce un argumento que convendría discutir, aunque sea brevemente. Admitiendo que la historia que hemos documentado y que queremos analizar sea una historia de éxito, chasta qué punto ha pesado en la misma, en sus logros y desarrollo, el peso de las personalidades implicadas y, especialmente, el peso de Giuliano Amato y Sabino Cassese? Se trata de una cuestión perfectamente legítima, no sólo porque es evidente que, después de todo, las personas, sus capacidades y sus orientaciones, constituyen un recurso esencial de todo proceso de cambio, sino porque sobre todo en este caso nos encontramos con personas muy particulares. Tanto Amato como Cassese gozan de una profesionalidad muy relevante respecto de la materia de la que nos ocupamos, personas que están en la cúspide de sus respectivas profesiones, y con una amplia experiencia internacional que les proporciona constantes puntos de referencia, a menudo por encima de los propios especialistas en cada tema. No obstante, considerar que la capacidad profesional de los "operadores" ministeriales de la reforma por el hecho de ser condición necesaria, sea ya condición suficiente, sería, desde mi punto de vista, al menos peligroso. Sólo deberíamos recordar que, ya en el pasado, el puesto de Ministro para la Función Pública había sido ocupado por una personalidad del relieve de Massimo Severo GIANNINI, sin que ello conllevase una aceleración de los procesos de reforma que fuera vagamente comparable con lo sucedido en el bienio 1992-94. Hacer recaer en el debe de GianNin el "fracaso" de entonces sería tan injusto como explicar todo lo que ahora ha sucedido a partir de la "capacidad" o del "activismo" de Amato y Cassese.

Otro elemento normalmente poco considerado en la literatura de procesos decisionales y que creo que merece una cierta atención es el factor tiempo. En relación a este concepto podemos considerar al menos dos acepciones. Una sería el tiempo en el que las reformas se han producido o, dicho de otra manera, las contingencias en las que se desarrolla el proceso decisional. En esta acepción la variable tiempo se identifica con el entorno externo (y sus transformaciones) a la política de reforma administrativa, y considerarla entre las variables explicativas significa simplemente afirmar que la reforma ha aparecido en ese período porque una serie de factores externos la estimulaban, o al menos no la obstaculizaban. Más interesante, en cambio, es la segunda acepción de la variable tiempo, que hace referencia al tiempo que los actores tenían a su disposición para llevar a la práctica sus intenciones. Aquí la cuestión que se plantea es la de preguntarse si el hecho de que "faltase tiempo", en el sentido de que ambas etapas gubernamentales eran (en medida diferente), claramente "a plazo fijo", significó una limitación o más bien una oportunidad para la reforma y su eficacia. La hipótesis que quisiera adelantar es sólo aparentemente paradójica, y pone el acento en la oportunidad que constituyó la falta de tiempo. En efecto, por un lado, esa falta de tiempo ha aumentado la intensidad de las preferencias de los protagonistas de la reforma, mientras, por otro lado, los ha llevado a concentrarse en aquellos aspectos que podían concluir, aunque fuera como propuesta, en 
el lapso de tiempo que tenían como Gobierno. Este argumento puede aplicarse sin duda de forma clara por lo que se refiere al Gobierno Ciampi y al ministerio Cassese, pero puede también quizás aplicarse al primer Gobierno y al menos asume en parte los dos sentidos más arriba indicados del factor tiempo.

En resumen, parece que haya existido una clara conciencia del hecho de que la "ventana" abierta entre el final de la estructuración precedente del poder político y el inicio de la operatividad del nuevo, se cerraría en lapso de tiempo breve, y ésa haya sido la razón por la que las operaciones llevadas a cabo han seguido esa pauta de reducción y de simplificación que hemos ya mencionado. Las incertidumbres sobre lo que ocurriría "después" eran tan altas que incluso los potenciales opositores de muchas de las transformaciones tenían dificultades para adoptar las típicas tácticas dilatorias, ante todo porque temían que las nuevas estructuraciones a lo mejor serían aún más perjudiciales para sus intereses, por lo cual la intensidad de sus preferencias decrecía en modo directamente proporcional al aumento de las preferencias de los promotores de la reforma.

Pero, quizás, el factor de éxito más importante lo constituyó la gran autonomia de que gozaron los dos gobiernos presididos respectivamente por Giuliano Amato y Carlo Azeglio Ciampi. Inevitablemente, la reforma de la Administración puede avanzar sólo si existe una decidida voluntad por parte del ejecutivo, y ello es más fácil cuando otros sujetos que podrían obstaculizar la transformación del statu quo son débiles, o al menos no están en condiciones de influir sustancialmente sobrê las opciones que se van tomando en el Consejo de Ministros. Esas condiciones se verificaron en el bienio 1992-94, y no pueden separarse de la crisis que en aquellos años estaba atravesando el sistema político (y que, para ser sinceros, no parece que hoy haya llegado a un punto estable de equilibrio).

Consideremos por separado los diversos elementos que conforman la afirmación anterior. Ante todo, no hay duda que es la crisis de los partidos políticos la que explica la ausencia de un conjunto de resistencias y obstáculos. Parece importante por dos motivos distintos: ante todo, porque la crisis del viejo sistema del pentapartito ${ }^{4}$ - pero también en parte la crisis de la oposición de izquierdas - rompió muchos de los ligámenes existentes con los grupos de intereses potencialmente hostiles a los cambios, y ha disminuido los recursos políticos de esos mismos grupos. En segundo lugar, porque el hecho de que los partidos no fuesen en realidad actores de primer nivel, y sin duda no fuesen protagonistas ni "responsables" de la política gubernamental, ha sido un factor importante en el abandono de la visión "sinóptica" de la reforma. En sentido contrario, puede verificarse empíricamente esa segunda percepción, analizando los casos en los que los partidos políticos sí han tenido influencia, aunque esa influencia consistiese básicamente en el veto a ciertas decisiones. Este fue el caso de la (frustrada) reforma de los Ministerios propuesta por Cassese y bloqueada por la oposición de los partidos. Esa reforma consistía en la reducción de las Administraciones centrales a través de la fusión de los Ministerios existentes, y es fácilmente comprensible que sólo podía operacionalizarse por parte de un Gobierno que estuviese próximo a la dimisión, aunque sólo sea por el hecho de que, una vez atribuida la distribución de responsabilidades de gobierno, sería muy difícil quitar a alguno de los neo-ministros una cartera ministerial que les acababa de ser asignada. En cambio, probablemente por un sentido de coacción genérico más que por un cálculo racional, se constató una gran oposición a una reforma que era vista como una expropiación realizada por un gobierno "no político" de una reforma de orden general que de alguna manera sobrepasaba sus atribuciones.

Un corolario de este elemento lo observamos en la escasa significatividad, y esto sí es una absoluta novedad en la Italia contemporánea, de la arena parlamentaria en la elaboración de los textos normativos. Un análisis de los cambios que realiza el Parlamento en los textos presentados por el Gobierno, muestra cómo las modificaciones introducidas fueron absolutamente marginales, y las entrevistas realizadas han demostrado que, al margen de la iniciativa de algún parlamentario a título individual, el paso tanto por la comisión como por el pleno ha sido prácticamente inocuo. A ese resultado contribuyó notablemente la adopción de la vía de la delegación legislativa, con los subsiguientes decretos legislativos que se sometían sólo a la opinión de las comisiones parlamentarias y que eran incluso posteriormente modificables por nuevos decretos legislativos posteriores. Fue éste un esquema de acción que, si bien es tradicional en materia de reforma administrativa (existían precedentes en la ley 382 y en el DPR 616), fue adoptado en la XI Legislatura precisamente para aumentar la autonomía del ejecutivo en un período de crisis política, económica, financiera e institucional.

En definitiva, ello ha sido posible hacerlo no a pesar, sino debido al vacio político. Lo que indica quizás nuevas posibilidades en el futuro para adoptar iniciativas aún más radicales.

Si pasamos ahora a examinar el comportamiento de los grupos de interés, y en particular del más importante de entre ellos, es decir, los sindicatos de empleados públicos, podemos constatar notables diferencias entre la primera y segunda fase en que se articula el período que estamos considerando. Lo común a ambos períodos es la debilidad relativa, al menos si la comparamos con los puntos de referencia anteriores de partidos y parlamentarios, del sindicalismo autónomo que de hecho logra, en solitario y en la primera fase, introducir excepciones a las reglas generales establecidas en materia de privatización de las relaciones laborales. Por lo que hace referencia al sindicato confederal, constituye ciertamente uno de los principales actores de las reformas Amato-Sacconi, sobre todo en lo referente a la voluntad de llegar a una completa contractualización de las relaciones laborales, que de hecho constituía una reivindicación sostenida a lo largo de más de diez años. En este caso, no se puede hablar tanto de autonomía del ejecutivo, como de concordancia de visiones e intereses entre responsables políticos y responsables sindicales. Muy diferente es el cuadro de referencia en la segunda de las fases que analizamos. En esta segunda fase encontramos verdaderos conflictos, muchas veces subterráneos pero no por ello menos efectivos, entre sindicatos y el ministro Cassese. Si tales conflictos no llegaron a constituirse en verdaderos obstáculos en la vía de las reformas, ello fue debido tanto al prestigio 
y la posición de privilegio de la que gozaba el gobierno en su conjunto (gracias asimismo a los acuerdos alcanzados con los sindicatos en julio de 1993), como a que el sindicato confederal estaba dividido internamente entre CGIL y las otras dos confederaciones, CISL y UIL, así como entre confederaciones y federaciones sectoriales.

Si analizamos ahora el papel de la burocracia en general y en particular el papel de los grandes cuerpos, las conclusiones a las que llegamos no son muy distintas de las hasta aquí contempladas. Conviene recordar que, en general, nuestra burocracia no brilla ciertamente por su activismo ni por su iniciativa. En esta situación de debilidad se inserta el hecho de que la exigencia de superar lo que se considera una situación deficiente está ampliamente difusa entre amplios estratos de los cuerpos burocráticos, y muy frecuentemente compartida por los mayores responsables. Las oposiciones que se manifestaron, sea la de la Intervención General, sea la de la Corte dei Conti (en manera especial, ya que veía afectada una de sus funciones centrales, como era la del control preventivo de legitimidad), fueron contrapesadas tanto por las mismas divisiones internas de esos sujetos, como por el hecho de que otros organismos sin apoyar explícitamente al gobierno, sí se manifestaron dispuestos a "jugar" según las reglas fijadas por el gobierno. Me refiero, por ejemplo, al Consejo de Estado, cuyos dictámenes no sólo no han impedido el avance de la reforma, a pesar de que en ocasiones han diluido un poco la fuerza innovadora de ciertas medidas, sino que sobre todo han respetado los contenidos y los plazos, contribuyendo así a una radical aceleración del ritmo de trabajo.

En definitiva, ha sido la debilidad o la parcial colaboración de los actores políticos, sindicales y burocráticos, la que ha contribuido a reforzar el ejecutivo y a garantizarle un nivel de autonomía absolutamente inusitado. A estos factores negativos se añaden dos factores positivos, la gran importancia con que asumieron la reforma los dos Presidentes del Consejo de Ministros, y el reforzamiento en la segunda fase que sufrió el proceso de reforma desde el punto de vista técnico y cognoscitivo en el Ministerio de la Función Pública, verdadera "cocina" de las innovaciones.

En relación al primero de estos dos puntos, poco cabe añadir, ya que no es necesario resaltar la centralidad del Presidente de Gobierno en una materia como la que estamos tratando. Según los testimonios que hemos recogido, Giuliano Amato estuvo directamente implicado en alguna de las transformaciones administrativas (sea por los ligámenes directos que tenía con las cúpulas sindicales, sea por su experiencia en gobiernos anteriores) y Carlo Azeglio Ciampi se mostró muy sensible desde el inicio a la necesidad de operar un cambio en el sentido en el que iba la reforma, proporcionando un apoyo constante, sobre todo en el Consejo de Ministros pero no sólo en él, al ministro Cassese.

El segundo punto es, en cambio, muy relevante, ya que el reforzamiento de los recursos técnicos y cognoscitivos del Ministerio de la Función Pública (condición esencial para "mantener el ritmo" de una producción legislativa y reglamenta- ria muy amplia), ha venido a través de la incorporación externa de la profesionalidad necesaria, no sólo ni especialmente vía la activación de una red de consultores senior externos (que es una vía, para así decirlo, ya tradicional en el policy making italiano), sino sobre todo constituyendo una importante Secretaría Técnica, compuesta de jóvenes estudiosos y funcionarios coordinados con algunas figuras de mayor experiencia científica y profesional. Todo ello, evidentemente coordinado y dirigido por un Ministro que no sólo se dedicaba totalmente al tema, sin "distracciones" políticas ni parlamentarias, sino que además representaba la verdadera memoria histórica del ministerio, ya que había colaborado desde hacia 25 años y desde diversas posiciones, pero siempre con gran relieve, con prácticamente todos sus predecesores. Debemos recordar que Cassese gozaba además de la ventaja en el momento de su nombramiento de llevar seis años como director de un gran proyecto de investigación, financiado por el Consiglio Nazionale della Ricerca, sobre la "Organización y Funcionamiento de la Administración Pública", que ha dado lugar a una amplísima cantidad de investigaciones y publicaciones sobre casi todos los campos de la Administración pública, no sólo italiana. La suma de todos estos factores definía una situación que, desde muchos puntos de vista, se podría considerar como óptima, en el sentido de una correcta estructuración de los aparatos de ayuda a los Ministros para facilitarles la tarea de formulación de las prioridades de policy. Pero, y debemos subrayarlo ya que constituye la otra cara de la moneda, la relación entre la estructura permanente del ministerio y la secretaría técnica, salvo pocas y honrosas excepciones, fue prácticamente inexistente, y de hecho sólo paliada a traves del ministro y de sus más estrechos colaboradores. De hecho también en este caso, la experiencia del gobierno precedente, y probablemente de todos los gobiernos anteriores que quisieron realizar innovaciones relevantes, era sólo diferente en cantidad, pero no en calidad, de lo que fue la gestión de Cassese. Así, el decreto 29/93 fue básicamente elaborado por un grupo de personas sustancialmente externo al ministerio. En definitiva, aparte de la estructura administrativa en sentido estricto, y aparte de excepciones individuales, el aparato ministerial en el curso de la Legislatura ha perdido algunas funciones y no ha ganado en centralidad en el proceso de policy making, en buena parte porque no estaba en condiciones de servir de apoyo en una serie de iniciativas que, probablemente, una gran parte de sus funcionarios consideraba objetivamente poco adecuadas, o incluso peligrosass.

Pero, ¿de dónde procedían estas innovaciones y propuestas? De hecho, éste es otro factor de éxito que nos interesa subrayar ya que, entre otras cosas, acerca las dos fases en que hemos dividido esta Legislatura. En realidad, en su mayor parte las propuestas existian ya con un buen nivel de elaboración y se habían ido acumulando en el período anterior. Salvo algunas excepciones y sin querer en absoluto desmerecer el esfuerzo, también creativo, que era necesario para traducir todo esto en una acción legislativa concreta y reglamentaria, los proyectos realizados han recalcado las líneas de propuestas precedentes. Así, en relación al DL 39/93 se puede citar una propuesta de delegación legislativa presentada por el ministro Cirino Pomicino 
y que fue discutida por el Parlamento, o los documentos que circulaban desde hacía tiempo en relación a la privatización de las relaciones laborales ${ }^{6}$, y lo mismo podemos decir en relación a procedimientos como el nuevo reglamento de contabilidad, con la introducción de nuevos impulsos a la informatización, o la normativa para la emisión de los permisos de conducir. Incluso en materias en las que no existían proyectos completos, ciertamente las realizaciones constituían la traducción práctica de viejas aspiraciones, como por ejemplo el tema de la autoridad para la informática, o la introducción del control de gestión. En resumen, entre los factores que han permitido el avance de la reforma podemos mencionar la capacidad de los nuevos responsables políticos para abrir cajones en los que encontrar aquellas propuestas y proyectos que sabían ya existentes, y que en muchos casos ellos mismos habían contribuido a crear.

Hemos ya mencionado anteriormente el último de los factores facilitadores del éxito, pero vale la pena volver sobre ello, para subrayar la absoluta centralidad de tal factor para explicar lo que ha pasado en el bienio 1992-94. En términos simples, lo que queremos decir es que ha sido la situación de emergencia financiera la que ha generado la conciencia difusa de proceder con urgencia en el sentido de la reforma administrativa. Las implicaciones de esta afirmación son dos, una de tipo positivo, otra de tipo negativo. Desde el punto de vista positivo, podemos afirmar que sin ese ligamen estrecho con los problemas de las finanzas públicas, hubiese sido casi imposible superar las muchas resistencias existentes, sobre todo en lo referente a la reducción organizativa y a la simplificación procedimental. En sentido negativo, es evidente que no podemos interpretar la reforma administrativa en clave de respuesta a la crisis política e institucional: en realidad, esa misma crisis política hubiera podido generar una situación en la cual los poderes reales hubiesen pasado a ser ejercidos directamente por las cúspides burocráticas existentes que, como hemos ya señalado, no parecían ser especialmente favorables a las reformas. En la práctica, es la combinación de estos dos factores lo que explica la forma a través de la cual el tema administrativo ha pasado de ser, en pocos meses, de un elemento ritual en todo programa de gobierno a uno de los focos centrales de la agenda gubernamental.

\section{Conclusiones: luces y sombras de la experiencia}

Ha llegado el momento de examinar el conjunto de la investigación realizada y de preguntarse si y en qué medida lo que ha ocurrido en la XI Legislatura autoriza a pensar que el proceso de renovación y modernización de la Administración italiana puede seguir adelante, en el futuro, con las mismas características que ha ido mostrando en el pasado.

Lo primero que hay que señalar es que el proceso realizado no es en absoluto irreversible, y que por otra parte es muy probable que si se deja que siga sus propios derroteros, tienda a sufrir momentos de retroceso o inversiones de rumbo. Como ya he señalado, la iniciativa hacia la renovación ha surgido casi totalmente desde el exterior de la maquinaria administrativa precisamente responsable de pensar y proyectar los procesos de reforma (es decir, el Ministerio de la Función Pública) y no muestra por tanto momentos de autoalimentación significativos. Por otra parte, uno de los centros neurálgicos de la Administración pública, la Intervención General del Estado, ha mostrado de forma clara su rotunda hostilidad a una transformación que enfatizase el proceso de descentralización y, por tanto, implicase la reducción del papel del control central y previo sobre la regularidad y legalidad de los actos administrativos.

Ni tan solo la centralidad que el tema conquistó en la agenda gubernamental y en los medios de comunicación presenta características de irreversibilidad. Sino por otros motivos, simplemente porque la atención política y periodística tiende a desplazarse de nuevo hacia la reforma de las "ramas altas" del sistema institucional ${ }^{7}$, volviendo a caer en el error tradicional según el cual la Administración, como los temas de intendencia, seguirá de forma automática la evolución que siga el orden constitucional. Pero, a pesar de todo, se puede advertir que ese período no ha pasado en vano, y que la mejor prensa (sobre todo la de contenido económico) ha adquirido una sensibilidad sobre la problemática administrativa que es imposible que pierda a corto plazo, pero es ése un resultado tan valioso como frágil.

$\mathrm{Si}$ a ello se añade que muchas de las condiciones de éxito puestas de relieve en el período anterior, y sobre todo aquellas que tiene que ver con la autonomía del ejecutivo, están condenadas, salvo transformaciones constitucionales, a ir disminuyendo en importancia, sino por otra razón, porque los grupos de interés tienden a reafirmar los ligámenes con el Parlamento cortados en la crisis 1992/94, la conclusión es que la vía por la que debe proseguir la obra iniciada muestra una pendiente pronunciada.

Pero, entrando ya en elementos sustantivos, ¿cuál es la valoración que podemos dar al conjunto de la actividad de reforma administrativa desarrollado por los gobiernos Amato y Cassese y que hemos intentado documentar en las diversas partes que componen esta investigación? ¿Podemos decir que en el período considerado se logró efectivamente "reinventar la Administración pública", para retomar el título de gran éxito en los Estados Unidos?

En líneas generales, creo que el juicio sólo puede ser altamente positivo, y para afirmar tal cosa, retomando afirmaciones ya realizadas, las razones principales son las siguientes:

1. La ya recordada capacidad de colocar el "tema administrativo" en la agenda politica y gubernamental y de atraer sobre ese tema el interés de los medios de comunicación. Y ello es así, a pesar de que, como ya hemos dicho, se trata de un éxito frágil y provisional. Pero, lo cierto es que lo ocurrido en la XI Legislatura demuestra de una vez por todas que es posible superar el retraso histórico de nuestro país en este sector, superando la retórica, y mostrando las ventajas de una aproximación "laica" y madura a una cuestión que no sólo condiciona la eficiencia del "sistema Italia", sino que condiciona la practicabilidad concreta de los proyectos de transformación institucional. 
2. La amplitud del ámbito de intervención propuesto y practicado, y sobre todo, la superación de una concepción globalista de la reforma a favor de una aproximación diferenciada y adecuada a las diversas necesidades de transformación. Si quisiéramos destacar cuál es el legado que la gestión Cassese del Ministerio para la Función Pública ha dejado a los gobiernos sucesivos, deberíamos referirnos al hecho de que reformar, modernizar y adecuar la Administración pública italiana quiere decir intervenir, con instrumentos y criterios diversos, en las muchísimas unidades organizativas que componen el sector público italiano.

3. La existencia de verdaderos elementos de discontinuidad con el pasado, de transformaciones, al menos potencialmente, no incrementalistas y capaces de operar transformaciones radicales en los modos de ser y de operar de la burocracia. Sin voluntad de ser exhaustivo, recordaremos algunos de estos elementos a modo de ejemplo:

* la introducción de la norma según la cual en una amplia categoría de situaciones no será necesario obtener autorización administrativa previa, siendo sustituida por una simple comunicación por parte del sujeto privado;

- la introducción de las Cartas de Servicios que incorpora la contractualización implícita entre prestadores de servicios y usuarios;

* la introducción del presupuesto en las universidades que responsabiliza a estas instituciones del propio gasto en materia de enseñanza, poniendo así las bases de una autonomía efectiva;

* la completa contractualización de las relaciones laborales en el sector público, a través de la capacidad derogatoria que los nuevos contratos tienen sobre la legislación posterior a la entrada en vigor del contrato precedente;

* la abolición de una gran parte de los controles previos de legalidad por parte de la Corte dei Conti y la introducción generalizada del control interno sobre los resultados de la gestión;

* la obligación impuesta a todas las Administraciones de medir las cargas de trabajo del personal a su servicio, que más allá de alguna ingenuidad en la definición de la metodología a utilizar, y sobre todo de un exceso de confianza en la suficiencia de las "normas correctas", tiende a prefigurar la superación de las plantillas orgánicas de carácter estático y a afirmar un rol del Ministerio para la Función Pública como agente de innovación, estímulo y control de los sistemas organizativos públicos;

* algunas de las nuevas instituciones creadas (como la Agencia para la contratación y la Autoridad para la informática) podrán desarrollar, si se sostienen adecuadamente, modalidades innovadoras en la gestión de las distintas problemáticas.
Estos "éxitos" generales y específicos no deben hacer creer que el cuadro final sólo esté compuesto por luces, sobre todo si comparamos la experiencia italiana con los intentos desarrollados en otros países. En realidad, existen límites de carácter general que conviene poner en evidencia con franqueza. Tres puntos, en particular, creo que debería subrayar:

1. Ante todo, el carácter casi totalmente legislativo o como mínimo normativo del tipo de intervención desarrollado; un ejemplo de esta insuficiencia lo constituye el tema del control de gestión, en el que, como apunta D. SORACE, "el problema más urgente ...no parece ser el desarrollar descriptivamente el tema del control de gestión en una ley, sino más bien de crear las condiciones para que un sistema de ese tipo pueda funcionar"; y, en cambio, las iniciativas de carácter diferente (por ejemplo, en el terreno de la formación y, al menos en parte, en los proyectos experimentales propuestos y realizados), aparecen como claramente subalternos a las transformaciones normativas y claramente también minoritarias desde un punto de vista cuantitativo; a pesar de que aún es pronto para valorar la eficacia de esas intervenciones, que en la gran mayoría de los casos no han concluido todavía, no creo que sea ir demasiado lejos si afirmo que estas iniciativas no parecen revestir una importancia excesiva; por otra parte, y teniendo en cuenta la inevitable viscosidad de los mecanismos de adecuación, conviene subrayar que, a unos meses de distancia de la aprobación de los textos legislativos y reglamentarios, en realidad existen sólo oficinas de relaciones con el público o sistemas de control de gestión, allí donde ya existían antes de las reformas, y constatar asimismo como la metodología elaborada en el interior del Ministerio para medir las cargas de trabajo ha sido abandonada en muchos casos a favor de métodos mucho más ágiles elaborados externamente. Como ya he apuntado anteriormente, falta aún un actor central en el proceso de reforma, sin que pueda considerarse como tal el Ministerio para la Función Pública, incluso después del nuevo decreto de organización.

2. En segundo lugar, conviene señalar la ausencia de iniciativas significativas en algunos terrenos cruciales y más en general la ausencia de algunas dimensiones importantes; y aunque pueda parecer casi paradóico, el primer elemento de juicio se aplica al tema de los directivos (un tema que probablemente constituye la clave de toda la reforma administrativa), lo que no puede ser contestado haciendo referencia a las previsiones del DL 39/93 (donde por cierto los llamados núcleos de valoración se encuentran sólo en sus inicios) en los principios generales, ni en el modo de reclutamiento (tampoco éste aún activado); entre las dimensiones casi absolutamente ausentes, en cambio, se puede recordar el tema de las autonomías territoriales que ha recibido, quizás por los motivos que mencionaré al final, una atención más bien escasa y poco significativa; y la lista podría continuar y debería incluir al menos (a pesar de las tentativas realizadas y los propuestas ya disponibles) la reforma radical de la contabilidad pública, que hoy por hoy impide el desarrollo de las esenciales autonomías organizativas de los diversos organismos; para todos estos problemas y para estas insuficiencias, existen, sin duda, buenas razones y quizás tampoco podía 
hacerse de forma distinta, pero nadie negará que la agenda de futuro resulta particularmente densa.

3. En tercer y último lugar, uno de los límites principales de las transformaciones realmente realizadas coincide, sólo en apariencia de manera paradójica, con uno de los factores de éxito que hemos recordado anteriormente, es decir, la disponibilidad de propuestas con un buen nivel de elaboración; es evidente, de hecho, que este elemento permite la eficiencia decisional, en el sentido de que ha sido posible pasar rápidamente de las palabras a los hechos, aunque sólo sea a efectos normativos, pero al mismo tiempo, ello genera una dependencia de las elaboraciones precedentes; y aquí conviene sin duda preguntarse si la concepción tradicional y neo-tradicional de la reforma administrativa resulta aún adecuada para el objetivo de construir la Administración del nuevo milenio ; y en este punto, mis dudas son importantes; es cierto que algunas de las mayores insuficiencias de la ideología de la reforma administrativa de los años 60 han sido superadas: por ejemplo, la confianza en los poderes salvadores de la planificación y de la coordinación formal ha sido superada, en los proyectos de reforma, a favor de una más realista y sobre todo más dinámica concepción que pone en el centro la descentralización (como subespecie del principio de subsidariedad) y la evaluación ex post de los resultados (como mecanismo de adaptación continua de las estructuras y los procedimientos a exigencias cambiantes; a pesar de todo ello, una gran parte de las propuestas de mayor relieve hacen aún referencia a la concepción neotradicional tal como la hemos caracterizado en las páginas precedentes; podemos, por tanto, preguntarnos, por ejemplo, si la transformación de los ministerios vía fusión de los ya existentes y la introducción generalizada de la figura de los secretarios generales no prefigura un cierto gigantismo administrativo gobernado por los niveles de staff en vez de la preeminencia deseable de los de línea; o incluso si es realmente posible articular la separación entre politica y administración distinguiendo sus funciones respectivas y no sólo como separación organizativa (lo que plantea el problema de los tipos de nombramiento y de garantía para las burocracias profesionales); o más aún, si la vía esencialmente jacobina seguida de forma tradicional y también de forma reciente, puede practicarse efectivamente a medio y corto plazo; 0 , más radicalmente, si no empieza a ser ya el momento de la superación de la "otredad" de la Administración pública que deriva del dominio del Derecho administrativo.

En definitiva, creo que el período de reforma que hemos atravesado, precisamente por su incisividad, demuestra la exigencia ineludible de iniciar una reflexión y una profundización que supere las insuficiencias de la tradición desarrollada por la comunidad de estudiosos y profesionales, en una amplísima parte juristas de formación, que hasta ahora y sin solución de continuidad en toda la historia de la Italia unitaria, han dominado absolutamente el marco de las reformas. Si hemos de tomarnos en serio la necesidad, no sólo italiana y no sólo motivada por el retraso de nuestros aparatos administrativos de "reinventar el gobierno y la Administración", necesitamos conseguir la construcción y la difusión de una nueva doctrina que funcione, como ha sucedido en el nacimiento del Estado liberal y después en la construcción del Estado de bienestar, como ideología de referencia para la amplia comunidad de científicos, políticos y burócratas.

Desde este punto de vista, con un poquito de provocación, podría afirmarse que uno de los primeros problemas que tenemos en el orden del día es básicamente de carácter cultural. Necesitamos liberarnos de una vez por todas del mito de la Administración francesa, sino por otros motivos, por el simple hecho de que esa Administración se basa en un sistema de selección de las élites dirigentes que no parece posible importar a nuestro país, tanto por la estructura económica y social, como por el sistema de formación que tenemos. En Italia no tenemos las grandes écoles, y no parece probable que logremos crearlas, al menos en lo que se refiere al sector público. En relación a la Administración francesa, a sus grands corps, y a sus escuelas, tenemos actualmente un retraso de al menos 200 años. Por otra parte, como muestra la literatura internacional, el modelo francés no es el único al que podemos referirnos, y más valdría, por tanto, empezar a pensar sobre lo que está sucediendo en Gran Bretaña y en los Estados Unidos, en vez de continuar sacando la mayoría de los ejemplos de la Administración gala.

Sea como fuere, relanzar un debate con un giro de 360 grados sobre el tema de la Administración pública es una exigencia ineludible. En primer lugar, porque el momento es propicio, ya que lo que está en discusión hoy no sólo en Italia y no sólo a causa de la crisis política e institucional que atravesamos, es la necesidad de una gran transformación del Estado y de la forma de gobernar. Las tendencias al federalismo y al presidencialismo, como decía al inicio, sólo podrán funcionar si se traducen en una nueva "forma de Administración", más capaz de garantizar la eficacia y la eficiencia de las políticas públicas. La organización del Estado liberal ha sido construida en definitiva a partir de la exigencia de garantías por parte de cada ciudadano en relación al poder, y la del Estado del bienestar, nunca cumplida del todo, a partir de la exigencia de asegurar un gobierno global (a través de la planificación y de la coordinación intersectorial) a la sociedad industrial. La pregunta que debemos plantearnos es la de cuál debe ser la organización adecuada al Estado pos-keynesiano y, por tanto, cuáles deben ser los principios que deben informarla. Me doy perfectamente cuenta que se trata de una pregunta gigantesca y que sería de locos imaginarse que sólo de una respuesta completa a estas preguntas podrán ser deducidas las necesidades efectivas de reforma. Pero no plantearse el problema significa exponer toda reforma y toda tendencia de transformación al peligro de la circularidad, o al peligro de ser saboteadas o diluidas por todos aquellos que tienen interés en el mantenimiento del statu quo, y que no comprenden en nombre 
de qué principios y en nombre de qué fines generales tendrían que renunciar a sus posiciones de poder y a sus privilegios.

Dicho de otra manera, reinventar la organización y los instrumentos de gobierno (el government inglés) no es posible, o al menos resulta extremadamente frágil, si no se afronta el problema de qué significa gobernar (la governance) ${ }^{8}$ y para qué fines generales debe desarrollarse. Reflexionar sobre estas cuestiones no me parece en absoluto un lujo, sino la verdadera misión (en el significado organizativo y no religioso del término) de la comunidad de los estudiosos?.

De hecho, podemos decir que existe un alto grado de consenso a nivel internacional sobre los criterios que deberían guiar la transformación de los aparatos y de los procedimientos administrativos. Sin voluntad de exhaustividad y sin introducir los argumentos que están en la base de cada uno de esos criterios, sí podemos intentar enumerar aquellos que podrían estar en la base de las reformas administrativas venideras:

1. Separación entre funciones politicas y funciones administrativas entendida como separación entre organizaciones explícitamente destinadas a la formulación y a la evaluación de las políticas públicas (los Ministerios) y las organizaciones responsables de la implementación de las políticas y más en general de todas las tareas de gestión activa.

2. Adopción resuelta del principio de subsidariedad, sea en la relación entre Estado, Región y autonomías locales, sea en la relación entre órganos centrales y órganos periféricos, sea en la relación entre sector público, sector non profit y sector privado.

3. Descentralización de las responsabilidades a favor de las organizaciones operativas o de linea, trasladando el gobierno de los recursos (humanos, financieros y tecnológicos) alli donde se utilizan realmente para producir servicios para los ciudadanos; adopción de un sistema de programación y de control basado sobre presupuestos globales confiados a la responsabilidad de los directivos respectivos; redefinición correlativa de las funciones de las organizaciones de staff (intervención, dirección del personal, etc.), en términos de organizaciones al servicio de las líneas operativas.

\section{Desarrollo de las funciones de gobierno politico y de dirección administrativa a todos los niveles como defi- nición de las misiones y de los objetivos de las unidades subordinadas y como sistema de gobierno del personal directivo de rango inferior.}

5. Correlativa desregulación de los mecanismos de gestión, manteniendo la procedimentalización sólo con la finalidad de la garantía hacia el exterior; consiguiente revalorización de la figura y de la responsabilidad de los directivos, a través de la eliminación de las limitaciones existentes a la plena posibilidad de gestión de recursos financieros y humanos asignados para el cumplimiento de sus objetivos.
6. Adopción generalizada de la evaluación y del control de resultados, como base fáctica para la negociación entre las organizaciones y los niveles de responsabilidad que conforman la Administración pública.

Si en esta lista podemos volver a encontrar alguno de los elementos de discontinuidad que ya hemos mencionado en relación a las reformas administrativas de la XI Legislatura no es casual. Significa simplemente que el tema no es sólo "qué hacer", sino sobre todo por qué hacerlo y también cómo es posible llevarlo a cabo.

Es en este último punto de vista donde aparece el problema, muy presente en la mente de los protagonistas de las recientes reformas, de encontrar "el conflicto de intereses" que está en la base del surgimiento y del mantenimiento del proceso de transformación. Como hemos ya mencionado, en el Estado liberal era la posición central del ciudadano (sub especie de bomo oeconomicus interesado en la libertad de acción). En el Welfare State eran probablemente las grandes organizaciones de los intereses aliadas a las burocracias profesionales, asimismo interesadas en el desarrollo de las posibilidades de intervención sobre los problemas públicos (el triángulo big business, big unions, big State). Y hoy, fuera de elementos retóricos, ¿cuál sería la respuesta a dar?

Probablemente sea éste el mayor interrogante que podamos plantearnos. ¿Cómo superar el "jacobinismo" que ha caracterizado en buena parte el proceso que hemos intentado documentar y comentar? No es sólo un problema a definir como negativo, es decir, como contrapuesto al exceso de centralismo que hemos sufrido, sino que puede plantearse en positivo, en el sentido de preguntarse cómo podemos iniciar un proceso de cambio autoalimentado, un círculo virtuoso que parece imprescindible para conseguir transformaciones no marginales en la forma de ser y de operar de una Administración pública que ofrece resultados claramente insatisfactorios a unos costes quizás ya no por más tiempo soportables. Tampoco podemos contentarnos con afirmar que el problema es el de transformar los valores y los planteamientos de la burocracia y, por tanto, de actuar esencialmente a nivel de cultura organizativa. Es verdad que las transformaciones son quizás más importantes y más duraderas, pero ello sólo es cierto a largo plazo, mientras que a breve plazo puede acabar generando en conflictos y fracasos.

Un primer paso, probablemente esencial, sería de orden metodológico: es necesario conseguir la "protección" de la reforma de la Administración (y también la Administración) de las interferencias externas. La primera de todas, la que viene de una política excesivamente sensible a las mediaciones de los micro intereses. Ello quizás podría lograrse introduciendo modificaciones constitucionales (por ejemplo, restringir la posibilidad de que el Parlamento pueda legislar en temas de organización de las Administraciones públicas y de empleo público). Pero esa 
condición metodológica siendo necesaria, ¿resulta suficiente? ¿No corremos el riesgo de iniciar procesos referenciales perversos? Las dudas son lícitas, pero las respuestas no son en absoluto claras.

Personalmente no tengo la solución, y soy incluso escéptico sobre el hecho de que exista sólo una solución a este problema. En realidad, en esta fase podría incluso ser suficiente introducir una serie de contradicciones y de conflictos potenciales en el interior de las organizaciones burocráticas, para verificar hasta qué punto surgen procesos de transformación y para valorar en qué dirección avanzan tales procesos.

Para acabar con una nota de optimismo y refiriéndome a lo sucedido en Italia, creo que puedo afirmar que el sector en el que esa dinámica se ha iniciado ya es el ámbito de los entes locales y de los municipios, sobre todo en los lugares en que se ha producido la reforma "constitucional", a través de la elección directa de los alcaldes y sobre todo después de la reforma fiscal que ha dado mucha mayor autonomía. Esos cambios se han incorporado a la transformación más tradicional creada por la ley 142/1990 (potenciación del gobierno municipal en relación al pleno, y sobre todo abolición de gran parte de los controles previos). De tal manera que todo ello ha puesto en marcha una serie de mecanismos de responsabilización que se refuerzan mutuamente y que podrían consentir, a medio plazo, una sustancial transformación de las formas de gobernar nuestras ciudades y nuestro territorio. Evidentemente, estos resultados positivos no son automáticos y dependen de las limitaciones que serán impuestas a los entes locales y a los recursos de que dispongan. De la misma forma, el desarrollo de las autonomías, las universitarias, de la enseñanza o de las cámaras de comercio, por ejemplo, son piezas de estos cambios que se avecinan.
De hecho, con las paradojas que se quieran, todo el proceso de reforma administrativa en Gran Bretaña, en Estados Unidos y en muchos otros países, puede leerse como un proceso de descentralización y de distribución de responsabilidades. La lección que nos llega de las experiencias internacionales es, de hecho, que si es cierto que no basta la reforma del Estado en un sentido regionalista para propiciar automáticamente procesos de transformación de las Administraciones, y si por otra parte, también es cierto que el estímulo para la transformación puede venir desde un ejecutivo fuerte e ideológicamente motivado (como nos demuestra la experiencia de Gran Bretaña), ello no es óbice para negar que el momento de reforma administrativa que atravesamos por doquier presenta claros síntomas de discontinuidad con el pasado, tanto remoto como próximo.

Era tradicional afirmar que la reforma administrativa tenía que ir en el sentido de superar la fragmentación del Estado, en el sentido de propiciar una mayor coordinación y una mayor coherencia de las actuaciones públicas, lo cual implicaba una centralización del tema, asegurando una unidad de mando y los mecanismos de conexión, hoy, y no por problemas de moda intelectual, el tema dominante parece ser el tema de la descentralización y del desplazamiento de la atención hacia la parte de abajo (los empleados y los directivos de línea) y hacia el exterior (los ciudadanos/clientes). Las reformas de este fin de siglo, en definitiva, se caracterizan por una aproximación descentralizadora, independientemente del hecho que ello forme parte del proceso de reforma del Estado, e independientemente del hecho que el estímulo y la dirección del proceso se desarrolle aún en el centro.

Es en esta dirección, creo, que debemos proceder, con mayor coraje y rapidez. Sintiéndonos reforzados del hecho que los reformadores de la XI Legislatura nos han demostrado que la vía de la reforma es sin duda transitable.

\section{Notas}

Traducción de Joan SuBIRATS.

* Bruno Dente, es profesor de Ciencia Política en la Universidad de Venecia. Es uno de los Directores de Investigación del Istituto di Ricerca Sociale de Milán. Es considerado el máximo especialista italiano en Análisis de Políticas Públicas y uno de los mejores conocedores de la Administración pública italiana. Ha publicado La politiche Pubbliche in Italia, Governare la Frammentazio$n e$, y Politiche Pubbliche e Pubblica Amministrazione. Es miembro del Consejo Editorial de la Revista Governance.

${ }^{1}$ Este texto constituye una versión parcialmente modificada de la ponencia introductoria al Congreso organizado por la Fondazione Agnelli los dias 2 y 3 de diciembre de 1994 en Turín, Congreso que llevaba por título Reinventar la Administración Pública. Un balance del pasado, una agenda para el futuro, y que contaba con ponencias de M. CAMmelu, S. CASSESE, G. Costa, G. FalCon, C. LavaCA, G. PEters, D. SORACE, J. Subirats y G. Vecch. El conjunto de materiales del Congreso han sido publicados por la Editorial de la Fondazione Agnelli con el mismo título. La traducción al castellano ha sido realizada por J. SUBIRATS.

${ }^{2}$ Quiero llamar la atención sobre el hecho de que toda la peripecia de la reforma administrativa en Italia ha ido articulando una comunidad formada por estudiosos y funcionarios, mancomunados a través de una cultura y de un lenguaje compartido. Veáse "La cultura amministrativa negli ultimi 40 anni" en Queste Istituzioni, n.75/76, abril-setiembre 1988; y también en la misma revista (n.79/80, julio-diciembre 1989), mi intervención titulada "Alla ricerca dei fattori strategici del processo di riforma".

${ }^{3}$ Veáse "Il sofisma della privatizzazione del pubblico impiego" en Rivista Italiana di Diritto del Lavoro, 1993, pp.287-317.

${ }^{4}$ Como es sabido, el autor se refiere a la coalición de partidos que dominó la escena política italiana desde finales de los cuarenta hasta principios de los noventa. Con la Democracia Cristiana como fuerza mayoritaria, formaban esa coalición el Partido Socialista, el Partido Liberal, el Partido Republicano y el Partido Socialdemocrático. Fuera de esa coalición, pero en el llamado arco parlamentario, encontramos el Partido Comunista como principal fuerza opositora de izquierdas, y el partido Movimiento Social Italiano, que no era aceptado como posible fuerza de coalición en cualquier hipotética combinación de gobierno (nota del traductor).

${ }^{5}$ Desde este punto de vista, la misma reorganización del Ministerio, que le proporcionaba amplias competencias en tema de organización y procedimien- 
to (si bien es cierto que invertía la tendencia del Gobierno Amato al crear organismos que se ocupaban de estas cuestiones, debilitando de hecho el peso del Ministerio), podía llegar a ser letra muerta si no se aseguraba con procedimientos posteriores el potenciamiento de los recursos humanos y cognoscitivos que proporcionaran "piemas" sobre las que caminar a los nuevas competencias adquiridas nomativamente.

${ }^{6}$ De hecho, muchas de las críticas que se levantaron en relación a la primera versión del DL 29/93, partían precisamente de la consideración de que ese Decreto era más "retrógrado" que las elaboraciones previas que circulaban desde hacía tiempo.

${ }^{7}$ El autor se refiere a los temas que después de la caída del gobiemo Ciampi han sido considerados como más relevantes, como el sistema electoral, la reforma en sentido federal del Estado, etc. (Nota del traductor).
${ }^{8}$ Tanto en estos dos últimos casos como en otros anteriores se mantienen en inglés o en otros idiomas, los que el autor mantiene en esas lenguas en el original italiano (nota del traductor).

${ }^{9}$ Poner el acento sobre este nexo entre cómo se gobierna y con qué finalidad, significa entender mejor la reforma británica, probablemente la más relevante que hemos visto recientemente. Esa reforma no sólo estaba motivada en términos de eficiencia y racionalización, sino que suponía un cambio sustancial en el modo de ser y de actuar del Estado, lo que en definitiva prefiguraba una sociedad diferente, y ciertamente una relación diferente entre Estado y sociedad; se puede estar de acuerdo en que no todos los aspectos de ese proceso de reforma nos parecen convincentes, pero no se puede negar que esa reforma ha tocado algunos de los aspectos fundamentales del sistema. 
